\title{
Improving the poor prognosis of oral squamous cell carcinoma
}

\author{
Encourage better recognition and earlier referral
}

In Britain, oral squamous cell carcinoma kills at least 1400 people each year; nearly two thirds of patients with this cancer will die of their disease. ${ }^{1}$ But this gloomy proportion does not convey the full picture. Early disease, where the tumour measures less than $2 \mathrm{~cm}$ (T1) and there is no metastatic disease, responds to simple, cheap, non-debilitating treatment and has an expected five year survival of $80 \% .^{2}$ The poor prognosis for oral cancer in Britain reflects the large proportion of patients who have advanced disease by the time they are referred for specialist treatment. ${ }^{3}$

Oral cancer commonly presents as an indolent ulcer. ${ }^{4}$ These malignant ulcers fail to heal, distinguishing them from the very common traumatic and aphthous ulcers that heal spontaneously within two weeks. The commonest sites of the disease, the mobile tongue, floor of the mouth, and inside of the cheek, are all readily visible. Most cases occur in people over 50 (and in men more frequently than women). ${ }^{1}$ Tobacco in all its forms is an important risk factor ${ }^{5}$; excess alcohol intake ${ }^{6}$, and chewing of betel quid ${ }^{7}$ have also been implicated.

The cancer is locally invasive and metastasises to regional lymph nodes in the neck. Distant metastasis is rare in life, but necropsy studies have shown a high incidence of micrometastases outside the head and neck. ${ }^{8}$ As many as one in six patients have synchronous or metachronous carcinomas at other sites in the pharynx or tracheobronchial tree. ${ }^{9}$

Oral squamous cell carcinoma has several features which should theoretically ensure its early detection. Unlike patients with other tumours, patients with oral cancer have a choice of two professionals (their dentist and their doctor) to consult for advice and diagnosis. Specific premalignant lesions such as white or red patches (leukoplakia and erythroplakia) exist. These can be monitored and treatment started before frank malignancy develops. The cancer is usually readily visible with a pen torch.

Delayed referral, however, is common and is related to three factors. Firstly, most patients with oral cancer have never heard of the disease before diagnosis. Many patients therefore regard their symptoms as innocuous and treat themselves for a long time before consulting their dentist or doctor. Secondly, dentists refer two fifths of patients with oral squamous cell carcinoma. ${ }^{40}$ Over the past 14 years increasing charges have been levied on dental patients who seek treatment under the NHS, and the free dental check up has been revoked by government. These measures have deterred the elderly and lower socioeconomic groups, who are particularly at risk of oral cancer, ${ }^{11}$ from attending dentists. Their cancers are therefore more likely to be advanced by the time they visit a dentist and are referred on to a specialist centre.

Finally, doctors sometimes fail to recognise the ominous signs of the malignant oral ulcer and therefore offer patients inappropriate treatment such as systemic antibiotics or topical steroids. ${ }^{1213}$ This problem will be perpetuated as only six British medical schools offer any formal teaching on mouth examination by oral and maxillofacial surgeons (despite the availability of 260 such consultants for teaching).

Strategies to improve the prognosis of a disease include preventive measures, screening at risk populations to detect and treat early disease, educational campaigns to alert the public and professionals to the disease, and advances in treatment. Although campaigns have succeeded in encouraging many adults in Britain to stop smoking, alarming numbers of adolescents are continuing to take up the habit. ${ }^{14}$ This is particularly distressing as there seems to be an increasing cohort of young people with an aggressive form of oral cancer. ${ }^{1516}$ Virtually no publicity has been given to the link between oral cancer and tobacco.

Studies in the United States ${ }^{17}$ and Sri Lanka have shown that dentists and primary health care workers can achieve reliable sensitivity and specificity when screening for oral cancer. Unfortunately, the rate of hospital attendance by patients identified in this manner was poor. ${ }^{18}$ The British working group recently concluded that opportunistic screening of patients attending dental surgeries is the most cost effective option in Britain. ${ }^{19}$

Over the past decade advances in surgical technique have enabled more radical resection to be combined with radical radiotherapy, with improved prognosis for advanced oral cancer. ${ }^{20}$ Studies on chemotherapy have also yielded some promising results. ${ }^{21}$

Screening programmes and advances in treatment are, however, unlikely to reduce the morbidity and mortality of oral cancer much in the short term. The best hopes for rapidly improving the prognosis in this disease lie in education and prevention. Firstly, a national educational campaign should be set up to alert the public to the existence, symptoms, and signs of oral squamous cell carcinoma and so increase the chance that patients will present with early, treatable disease. The campaign should emphasise that all patients with unexplained mouth ulcers of more than three weeks' duration or intraoral red or white patches should attend the oral surgical department of their local hospital urgently. This 
campaign should also target teenagers and emphasise that smoking is an important cause. Tobacco advertising should be banned.

Secondly, a short course of teaching by oral surgeons should be introduced into the curriculums of all medical schools so that doctors, who are responsible for referring $40 \%$ of patients with oral cancer, are educated in examination and diseases of the mouth at the beginning of their careers. Finally, the free dental check up should be reintroduced, as recommended by the House of Commons Select Committee on Health, ${ }^{22}$ so that casual patients with oral ulcers have no financial disincentives to attending a dentist for diagnosis.

The treatment of advanced oral cancer entails long, costly surgical operations and prolonged courses of radiotherapy and chemotherapy. The disease puts an immense emotional and physical strain on patients and their families. Now is the time for the government and the Department of Health to institute the measures outlined above in a nationally coordinated campaign to reduce the burden of this crippling disease.

IAIN L HUTCHISON

Consultant oral and maxillofacial surgeon

St Bartholomew's Hospital,

London EC1A 7BE

1 Hindle I, Nally F. Oral cancer: a comparative study between 1962-67 and 1980-84 in England and Wales. Br Dent f 1991;170:15-20.
2 Evans SJ, Langdon JD, Rapidis AD, Johnson NW. Prognostic significance of STNMP and velocity of tumour growth in oral cancer. Cancer 1982;49:773-6.

3 Stell PM, McCormick MS. Cancer of the head and neck: are we doing any better? Lancet 1985 ;i:1127.

4 Scully C, Malamos D, Levers BG, Porter SR, Prime SS. Sources and patterns of referrals of oral cancer: role of general practitioners. $B M Y$ 1986;293:599-601.

5 International Agency for Research on Cancer. IARC monographs on the evaluation of the carcinogenic risk of chemicals to humans. Tobacco Smoking. Lyon: IARC, 1986 (vol 38).

6 International Agency for Research on Cancer. IARC monographs on the evaluation of the carcinogenic risks of chemicals to humans. Alcohol Drinking. Lyon: IARC, 1988 (vol 44).

7 International Agency for Research on Cancer. IARC monographs on the evaluation of the carcinogenic risk of chemicals to humans. Tobacco habits other than smoking; betel-quid and areca nut chewing; and some related nitrosamines. Lyons: IARC, 1985 (vol 37).

8 O'Brien PH, Carlson R, Steubner EA jr, Staley CT. Distant metastases in epidermoid cell carcinoma of the head and neck. Cancer 1971;27:304-7.

9 Carr RJ, Langdon JD. Multiple primaries in mouth cancer-the price of success. $\mathrm{Br} f \mathrm{Oral}$ Maxillofac Surg 1989;27:394-9.

10 Elwood JM, Gallagher RP. Factors influencing early diagnosis of cancer of the oral cavity. Can Med Assoc $\mathcal{f}$ 1985;133:651-6.

11 Townsend P, Phillmore P, Beattie A. Health and deprivation. Inequality and the North. London: Cross Helm, 1988:134.

12 Guggenheimer J, Verbin RS, Johnson JT, Horkowitz CA, Myers EN. Factors delaying the diagnosis of oral and oropharyngeal carcinomas. Cancer 1989;64:932-5.

13 Schnetler JFC. Oral cancer diagnosis and delays in referral. Br 7 Oral Maxillofac Surg 1992;30; 210-3.

14 Department of Health. The health of the nation. One year on. London: Department of Health, 1993:75.

15 MacFarlane GJ, Boyle P, Scully C. Rising mortality from cancer of the tongue in young Scottish males. Lancet 1987;ii:912.

16 Son YH, Kapp DA. Oral cavity and oropharyngeal cancer in a younger population. Review of literature and experience at Yale. Cancer 1985;55:441-4.

17 Moore C, Greenberg RA, Kane L. Feasibility study of a head and neck (upper aero-digestive tract) cancer examination. I Natl Cancer Inst 1987;79:409-15.

18 Warnakulasuriya S, Ekanayake A, Stjernsward J, Pindborg J, Sivayoham S. Compliance following referral in the early detection of oral cancer and precancer in Sri Lanka. Community Dent Oral Epidemiol 1988;16:326-9.

19 Community Dental Health. Screening for oral cancer and precancer: a report of a UK working group. 1993;10(suppl 1) 1-89.

20 Franceschi D, Gupta R, Spiro RH, Shah JP. Improved survival in the treatment of squamous cell carcinoma of the oral tongue. Am $\mathcal{F}$ Surg 1993;166:360-5.

21 Tobias JS. Has chemotherapy proved itself in head and neck cancer? $\mathrm{Br} F$ Cancer 1990;61:649-51. 22 House of Commons Health Committee. Fourth report: dental services. Vol 1. London: HMSO, 1993.

\section{Asymptomatic infection with hepatitis $\mathrm{C}$ virus}

\section{Screening of blood donors is detecting people with unsuspected, but dangerous infection}

The history of infectious hepatitis illustrates the everchallenging battle between humans, micro-organisms, and disease. In the 1970 s the organisms causing viral hepatitis types $\mathbf{A}$ and $\mathbf{B}$ were identified, allowing more accurate diagnosis and treatment, and the battle seemed to be on the way to being won. But nature persisted, and the term non-A non-B hepatitis was coined for the viral liver disease not due to hepatitis A or B virus, cytomegalovirus, or Epstein-Barr virus. Without specific assays the diagnosis of non-A non-B hepatitis was based on exclusion. The disorder accounted for $75-90 \%$ of cases of post-transfusion hepatitis, but it also included enterically transmitted, sporadic, endemic, and community acquired disease. ${ }^{1}$ In developed countries this type of hepatitis is second only to alcohol as a cause of chronic liver disease, cirrhosis, and hepatocellular carcinoma, but its treatment remains problematic. ${ }^{2}$

Most non-A non-B hepatitis is due to a single virus, hepatitis $\mathrm{C}$ virus, and with cloning of its virion, a positive single stranded RNA molecule, ${ }^{3}$ the diagnosis of liver disease became more reliable. Further precision came with assays for specific antibodies to hepatitis $\mathrm{C}^{4}$ and, more recently with identification of hepatitis $C$ virus RNA in the serum by reverse transcriptase polymerase chain reaction-a marker for viraemia and the infectious state. ${ }^{5}$

Three papers in this issue ( $\mathrm{p} 695,696,697$ ) present some of the important current issues in relation to an even more difficult problem-that of asymptomatic hepatitis C. ${ }^{6-8}$ Ryan et al found that many asymptomatic "healthy" people with antibodies to hepatitis $C$ virus are detected during screening of blood donors (obligatory since 1991), screening of relatives of patients with known hepatitis $\mathrm{C}$, and screening by health clinics. Some people with antibodies to hepatitis $\mathrm{C}$ virus who seem healthy may, in fact, have raised serum transaminase activities - as do patients with chronic hepatitis C.

Serum markers for liver disease may be normal in healthy people whose serum contains antibodies to hepatitis $C$ virus, but evidence is growing that most of these "healthy" people have histologically abnormal livers. Irving et al found that all but two out of 52 "healthy" blood donors infected with hepatitis $C$ virus had abnormal liver biopsy tissue. ${ }^{8}$ The abnormalities ranged from fatty change, chronic persistent hepatitis, chronic active hepatitis to cirrhosis. These were more severe in men and correlated with peak circulating alanine aminotransferase activities, suggesting the people with repeatedly positive results on hepatitis $\mathrm{C}$ virus testing must have their liver pathology assessed by biopsy. Follow up of such people is essential if morbidity and mortality from liver disease is to be averted.

Several issues must now be addressed. Should further screening programmes be planned to pick up more of these "healthy" people in the community, since they may be at risk of future liver disease by a synergistic effect with other agents such as alcohol and drugs (paracetamol, aspirin)? Established chronic hepatitis $\mathrm{C}$ is an insidious disease, and its progress is usually monitored by repeated measurement of transaminase activities and hepatitis C virus RNA.' It is difficult to recognise because it has a mild or subclinical course, clinical signs are often absent, and the transition from the acute to the chronic stage is silent. ${ }^{67}$ Once the condition has been diagnosed in someone who seems healthy, with or without abnormal results of liver enzyme tests, a liver biopsy seems essential. Subjects with an abnormal liver may progress to cirrhosis or hepatocellular carcinoma ${ }^{10}$ and so may warrant treatment. 